UMH-MG 01-01

\title{
Scalar perturbations in a primordial inflationary scenario
}

\author{
Júlio C. Fabriș \\ Departamento de Física, Universidade Federal do Espírito Santo, 29060-900, Vitória, \\ Espírito Santo, Brazil \\ and \\ Philippe Spindel[ \\ Mécanique et Gravitation, Université de Mons-Hainaut, 7000 Mons, Belgium
}

\begin{abstract}
We compute the spectral index for scalar perturbations generated in a primordial inflationary model. In this model, the transition of the inflationary phase to the radiative era is achieved through the decay of the cosmological term leading a second order phase transition and the characteristics of the model allow to implement a set of initial conditions where the perturbations display a thermal spectrum when they emerge from the horizon. The obtained value for the spectral index is equal to 2 , a result that depends very weakly on the various parameters of the model and on the initial conditions used.

PACS number(s): 04.20.Cv., 04.20.Me
\end{abstract}

\section{Introduction}

The big-bang standard model, based on the Friedmann-Lemaitre-Robertson-Walker (FLRW) solutions of Einstein's equations [1], suffers from some drawbacks which leaded cosmologists to propose the so-called inflationary models [2]. The common element to all these models is an exponential expansion phase of the size of the Universe prior to the beginning of the standard scenario that describes the adiabatic era extending from temperatures of

\footnotetext{
${ }^{1}$ e-mail: fabris@cce.ufes.br

${ }^{2}$ spindel@umh.ac.be
} 
order of $10^{-4}$ at Grand Unification era (GU) until $10^{-32}$, today's temperature, in Planck's units (which are the units that will be used throughout all this work).

From a kinetic point of view, the exponential expansion phase corresponds to a de Sitter geometry [3] which later on will transform itself into a FLRW geometry. From a physical point of view, this scenario raises (at least) two questions. What is the nature of the effective cosmological constant during the de Sitter phase of the history of the Universe? What is the mechanism which leads to the end of this phase? Among the different inflationary models, the first one to be proposed [1] looks specially interesting. It consists of a primordial inflationary model describing the birth and grow of the Universe as the result of a cooperative mechanism of zero total energy, generated by a quantum fluctuation occurring in a flat background. The idea that a quantum fluctuation may be the initial seed for the birth of the Universe has first been proposed by Tryon in 1973 [5]. It is because the total hamiltonian of gravity coupled to matter is zero (an important consequence of the invariance of general relativity with respect to time reparametrisation) and the energy contribution of the gravitational conformal modes is negative that it is possible to obtain non-trivial semi-classical solutions of Einstein equations where gravity and matter emerges from an empty flat space in a cooperative process.

In the model proposed by Brout et al. [4], matter is described phenomenologically through the action of a scalar field non-minimally coupled to gravity. A consistent asymptotic solution of the field equations [6, 7] describes a de Sitter geometry where the role of the cosmological constant is played by the (constant) density of the created matter which has, due to the energy conservation law, the characteristic equation of state of a cosmological constant $\rho_{\Lambda}+p_{\Lambda}=0$. On the other hand, the consistency of the model implies that the (effective) cosmological constant $\Lambda \equiv 8 \pi \rho_{\Lambda}$ is a free parameter of the obtained solutions. It must not necessarily be of order one, what justify a posteriori the semiclassical treatment employed. However, the mass of the scalar quanta fields is very large [6]: $M \simeq 6 \sqrt{\pi}$, this value being almost insensible to the value of the cosmological constant 8] even when the vacuum polarization effects are taken into account [9]; these effects play no significative role in the domain of validity of the model $(\Lambda<<1)$. On the other hand, the high value of the mass of the quanta is the signal that gravitational strong coupling must play an important role in the physics of this problem 10. The scalar field used in the model must be interpreted as a phenomenological description of complex objects, like black holes [11]. This interpretation, by the way, is confirmed by the analysis of the renormalization of the gravitational constant [10]. Indeed, the renormalized gravitational constant becomes singular [12] exactly for the critical value of the mass field $M=6 \sqrt{\pi}$, what is the origin of the analytical solution [13] of the semi-classical equations which describes, for this special value, the transition from a flat space to a singularity. Since the cosmological "constant" in this model is described a gas of black holes, their subsequent evaporation through the Hawking radiation mechanism may imply a time-decreasing of this effective cosmological "constant", generating hot radiation in the Universe.

For the moment, there is no consensus about how the inflationary phase ends. However, it is possible to distinguish, from the phenomenological point of view, essentially two types of transition to an adiabatic era: a first order phase transition, employed in the so-called old and new inflationary models, which deals with an effective cosmological 
constant given by the energy of a scalar field; a second order phase transition, as it be discussed here, based on a variable cosmological "constant".

The evaporation of the black holes or the infra-red fluctuations of the gravitational conformal modes [14] being responsible for the instability of the de Sitter geometry, it seems important to extract observational consequences of the model described above through the analysis of a simple scenario. In [15] analytical solutions of the FLRW type for the phenomenological model described above were obtained for any value of the spatial curvature $(k=0, \pm 1)$. They depend on four physical parameters: the initial value of the cosmological constant, $\Lambda_{0}$, its residual value $\Lambda_{\infty}$, the initial density of radiation in the Universe $\sigma_{0}$ and the characteristic time of transmutation of the cosmological constant into radiation energy $\tau$. The choice of a decaying of the cosmological constant into pure radiation is motivated in two ways. From the physical point of view, the temperatures encountered in this model permit us to ignore the masses of the usual degrees of freedom. From the mathematical point of view, the background model is exactly integrable and their perturbations easily computable.

Our purpose in the present work is to study the evolution of scalar perturbations in the inflationary model described before. We will restrict ourselves to the case $\Lambda_{\infty}=\sigma_{0}=0$ and $k=0$. These hypothesis lead to the advantage that approximate solutions to the perturbed equations can be easily obtained without to oversimplify the physics. Our model differs essentially in two ways from the usual inflationary models. First (after having fixed the residual gauge freedom allowed by the coordinate transformations), the perturbation equations in the framework of this two fluid model (the effective cosmological constant and radiation) will result in a third order perturbed equation (analytically solvable in the radiation regime and that otherwise can very well be approximated by a $2+1$ system of decoupled equations, also analytically solvable). The second characteristic of the model we discuss here concerns the nature of the initial spectrum of the fluctuations. As it will be verified later, the fluctuations evolve freely, i.e., they are determined by the Einstein's equations, only from the moment where the temperature of the created radiation is significative. Consequently, we will adopt as initial fluctuation spectrum a thermal spectrum, instead of a (hypothetical) spectrum inspired by quantum fluctuations of a vacuum state (a choice imposed more by metaphysical $]^{3}$ than by physical considerations; see however the discussions in [17, 18], for instance).

This paper is organized as follows. In section 2 , the field equations and the solution for the background are reminded. In section 3 the perturbed quantities are settled out, and approximate solutions presented. In section 4 , the spectrum of perturbations is discussed. Section 5 is devoted to the conclusions.

\footnotetext{
${ }^{3}$ During the fifties, Bertrand Russell observed: "The accusation of metaphysics has become in philosophy something like being a security risk in the public service. ... The only definition I have found that fits all cases is: 'a philosophical opinion not held by the present author'." 16.
} 


\section{The field equations}

In order to be complete, let us review briefly the solutions that will be used in our perturbative study. The geometry of the Universe is supposed to be of the type flat FLRW:

$$
d s^{2}=-d t^{2}+a^{2}(t)\left(d x^{2}+d y^{2}+d z^{2}\right) .
$$

This metric is a reasonable simplification for the primordial stages of the Universe. Moreover, in our case, due to the existence of an inflationary phase, the curvature can also be neglected in later stages of the evolution of the Universe. The unperturbed material source of the Einstein's equations is the sum of two comoving fluids:

$$
T_{\nu}^{\mu}=(\stackrel{0}{\rho}+\stackrel{0}{p}) \stackrel{0}{u^{\mu}} \stackrel{0}{u}_{\nu}+\stackrel{0}{p} \delta_{\nu}^{\mu} \equiv T_{(\text {rad. }) \nu}^{\mu}+T_{(\cos .) \nu}^{\mu}
$$

with $\stackrel{0}{\rho}=\rho_{(\text {rad. })}+\rho_{\Lambda}, \stackrel{0}{p}=p_{(\text {rad. })}+p_{\Lambda}$. The terms representing the radiation perfect fluid $\left(\rho_{(\text {rad. })}, p_{(\text {rad. })}\right)$ are supposed to represent the high energy matter degrees of freedom under the form of radiation:

$$
\rho_{(\text {rad. })}=\nu \frac{\pi^{2}}{30} T^{4}
$$

where $T$ is the temperature and $\nu$ is the effective number of degrees of freedom that we will take as being those of the standard model $(\nu=106.75)$, this value of $\nu$ constituting, of course, a lower bound at the energy considered. The term $\rho_{\Lambda}=\Lambda(t) / 8 \pi=-p_{\Lambda}$ is purely phenomenological. It represents the density of black holes created during the cosmogenesis phase and that evaporate later. We will assume it to be of the form :

$$
\Lambda(t)=\Lambda_{0} e^{-t / \tau}
$$

where the parameter $\tau$ is the characteristic time of the black hole evaporation which is of the order of the lifetime of the created black holes :

$$
\tau \sim \frac{2560 \pi}{\nu} M_{b h}^{3} \approx 10^{5}
$$

The equation of state of radiation, $\rho_{(\mathrm{rad} .)}=3 p_{(\mathrm{rad} .)}$, permits to obtain a linear second order differential equation for the variable $Z=a^{2}$ (a dot representing a derivative with respect to the cosmological time $t$ ):

$$
\ddot{Z}-\frac{4}{3} \Lambda_{0} e^{-t / \tau} Z=0
$$

The solution of this equation is given by a combination of modified Bessel functions:

$$
Z=\alpha K_{0}\left(\varpi e^{-\xi}\right)+\beta I_{0}\left(\varpi e^{-\xi}\right)
$$

where $\xi=\frac{t}{2 \tau}$ and $\varpi=\sqrt{\frac{16}{3} \tau^{2} \Lambda_{0}}$. The coefficients $\alpha$ and $\beta$ are given by

$$
\alpha=\varpi\left(I_{0}^{\prime}(\varpi)+I_{0}(\varpi)\right) \quad, \quad \beta=-\varpi\left(K_{0}^{\prime}(\varpi)+K_{0}(\varpi)\right) \quad \text {, }
$$


and are fixed by the conditions $Z(0)=1$ and $\dot{Z}(0)=+\sqrt{4 \Lambda_{0} / 3}$.

Notice that there was an error of sign in the ref. [9]. The scale factor written there corresponds to $\dot{Z}<0$, and the plateau appearing in the graph of the entropy as function of time is due to the crossing of the singularity $Z=0$. The graphs for the entropy and temperature as functions of time, for initial conditions (8) corresponding to an expanding Universe, are shown on figures 1 and 2 .

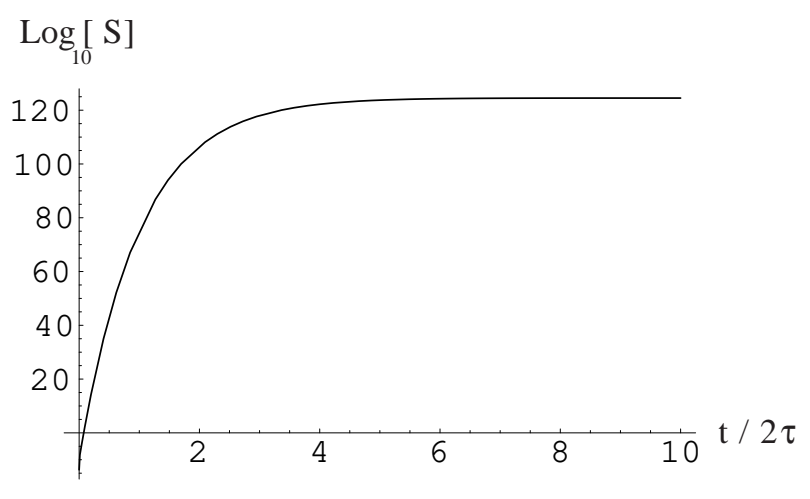

Fig. 1 Logarithmic plot of the radiation entropy as function of time for $\Lambda_{0}=5.510^{-7}$ i.e. $N_{e}=80$.

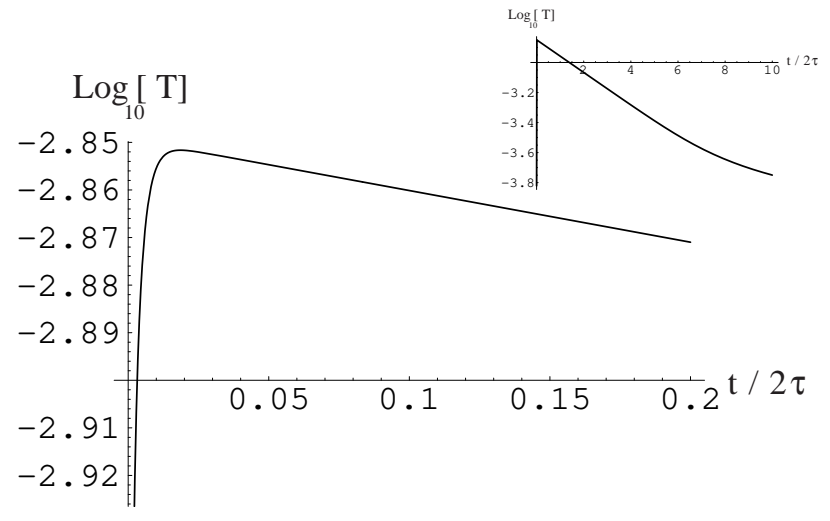

Fig. 2 Logarithmic plots of the radiation temperature as function of time for $\Lambda_{0}=5.510^{-7}$ i.e. $N_{e}=80$.

\section{The perturbed equations}

The perturbed equations for the scalar modes around the background solutions exhibited above are obtained through the standard methods. We suppose the metric perturbations

$$
g_{\mu \nu}(t, \vec{x})=\stackrel{0}{g}_{\mu \nu}(t)+\stackrel{1}{g}_{\mu \nu}(t, \vec{x})
$$

to be synchronous, i.e., such that $\stackrel{1}{g}_{\mu 0}=0$. The perturbations in density and velocity read

$$
\rho(t, \vec{x})=\stackrel{0}{\rho}(t)+\stackrel{1}{\rho}(t, \vec{x}) \quad, \quad u^{\mu}(t, \vec{x})=\stackrel{0}{u^{\mu}}+\stackrel{1}{u}^{\mu}(t, \vec{x}) \quad,
$$

with $\stackrel{0}{u}^{\mu}=(1,0,0,0)$. All perturbed quantities can be expressed through a Fourier decomposition

$$
\begin{aligned}
\stackrel{1}{g}_{i j}(t, \vec{x}) & =\frac{1}{\pi^{3 / 2}} \int h_{i j}(t, \vec{n}) e^{-i \vec{n} \cdot \vec{x}} d^{3} \vec{n}, \\
\stackrel{1}{\rho}(t, \vec{x}) & =\frac{1}{\pi^{3 / 2}} \int \delta \rho(t, \vec{n}) e^{-i \vec{n} \cdot \vec{x}} d^{3} \vec{n} \\
\stackrel{1}{u}_{\mu}(t, \vec{x}) & =\frac{1}{\pi^{3 / 3}} \int \stackrel{1}{u}_{\mu}(t, \vec{n}) e^{-i \vec{n} \cdot \vec{x}} d^{3} \vec{n}
\end{aligned}
$$


with $\vec{n} \cdot \vec{x}=\sum_{i} n^{i} x^{i}, \vec{n}$ being the comoving wave vector of the perturbations.

Introducing those perturbed quantities into Einstein's equations, we obtain three coupled perturbed equations:

$$
\begin{array}{r}
\left(a^{2} \dot{h}\right)^{\cdot}=a^{2} \delta \rho, \\
\left(a^{4} \delta \rho\right)^{\cdot}+(\rho+p) a^{4} \theta=8 \pi(\rho+p) a^{4} h \\
\left((\rho+p) a^{5} \theta\right)^{\cdot}=\frac{n^{2}}{3} a^{3} \delta \rho,
\end{array}
$$

where $\theta=\vec{n} . \vec{u}$ and $h=-16 \pi a^{-2} \sum_{i} h_{i i}$. The system of equations (14, 15, 16) is underdetermined since $\rho=\rho_{(\mathrm{rad} .)}+\rho_{\Lambda}$ and consequently $\delta \rho=\delta \rho_{(\mathrm{rad} .)}+\delta \rho_{\Lambda}$. We need a specific equation for the effective cosmological constant. Hence, we assume

$$
\left(\nabla_{\mu} T_{(\text {cos. })}^{\mu \nu}\right) u_{\nu} \equiv-\nabla_{\nu}\left(\rho_{\Lambda} u^{\nu}\right)-p_{\Lambda} \nabla_{\nu} u^{\nu}=\tau^{-1} \rho_{\Lambda}
$$

which reduces to $u^{\nu} \partial_{\nu} \rho_{\Lambda}=\tau^{-1} \rho_{\Lambda}$, since $\rho_{\Lambda}=-p_{\Lambda}=\Lambda(t) / 8 \pi$. For adiabatic perturbations $\left(\delta \rho_{\Lambda}+\delta p_{\Lambda}=0\right)$, it results ( $\tau$ being supposed to be a constant given by the microphysics)

$$
\stackrel{1}{u}^{\mu} \partial_{\mu} \stackrel{0}{\rho_{\Lambda}}+\partial_{t} \stackrel{1}{\rho}_{\Lambda}=\tau^{-1} \stackrel{1}{\rho}_{\Lambda}
$$

Since $\stackrel{10}{u}^{0}=0$, we obtain $\stackrel{1}{\rho}_{\Lambda}=L(\vec{x}) e^{-t / \tau}$. But this solution is a coordinate artifact. Indeed, the coordinate conditions $g_{\mu 0}=0$ yet remain satisfied when the residual infinitesimal transformations generated by the vector fields

$$
\epsilon_{0}=\phi(\vec{x}) \quad, \quad \epsilon_{i}=\psi_{i}(\vec{x}) a^{2}-\partial_{i} \phi(\vec{x}) a^{2} \int \frac{d t}{a^{2}}
$$

are performed. The choice $\phi(\vec{x})=\frac{\tau L(\vec{x})}{\Lambda_{0}}$ allows to make $\delta \Lambda(t)=0$. Notice that, in this way, the equations (14,15,16) are now gauge invariants with respect to the remaining coordinate transformations. Indeed, residual transformations $\epsilon_{0}=0, \epsilon_{i}=\psi_{i} a^{2}$ only modify $h_{i j}(t, \vec{x})$ up to a term whose time dependence is given by $a^{2}$; hence, $h$ is defined up to a constant, leaving $\dot{h}$ invariant. In other words, in the synchronous gauge $g_{0 \mu}=0, \delta \rho_{\Lambda}$ may be fixed to zero and all solutions of the perturbed equations (14,15, 16) now acquire a physical meaning, in contrast to what happens in the framework of a one-fluid model. In this later case, the third order perturbed equation may be reduced, using the residual coordinate freedom, to a second order equation. Here, we face a third order equation whose three integration constants have physical meaning.

From now on, we introduce the new set of variables

$$
\mathcal{T}=\left(\rho_{(\text {rad. })}+p_{(\text {rad. })}\right) a^{5} \theta \quad, \quad \mathcal{R}=a^{4} \delta \rho_{(\text {rad. })} \quad, \quad \mathcal{H}=a^{2} \dot{h},
$$

which permit us to write the system of equations (14, 15, 16) as

$$
\begin{aligned}
\dot{\mathcal{H}} & =\frac{\mathcal{R}}{a^{2}}, \\
\dot{\mathcal{R}} & =-\frac{\mathcal{T}}{a}+8 \pi\left(\rho_{(\text {rad. })}+p_{(\text {rad. })}\right) a^{2} \mathcal{H}, \\
\dot{\mathcal{T}} & =\frac{n^{2}}{3} \frac{\mathcal{R}}{a} .
\end{aligned}
$$


The solutions of this system and their cosmological implication will be the subject of the next section.

\section{Evolution of perturbations}

We are not interested here in the general solution of the system of equation (21-23) governing the evolution of the perturbations, for arbitrary values of their parameters. We shall only focus on the values of the parameters that are relevant for the cosmological model we consider here. In order that an inflationary model is acceptable, the scale factor must be amplified at least by 70 e-folds, before the Universe reaches the GU temperature $T_{G U}=10^{-4}$ where radiative era takes place. At this moment, the expansion of the Universe is described by the asymptotic expression

$$
Z_{a s}=\alpha \frac{t-t_{s}}{2 \tau} \equiv \alpha\left(\xi-\xi_{s}\right) \quad, \quad \xi_{s} \equiv \frac{t_{s}}{2 \tau} \simeq \ln \frac{\varpi}{2}+\gamma \quad,
$$

$\gamma \approx 0.577$ being the Euler-Mascheroni constant. Using the Einstein's equation $G_{00}=$ $8 \pi T_{00}$, we obtain

$$
\xi_{G U}-\xi_{s}=\left(\frac{128 \pi^{3} \tau^{2}}{90} \nu T_{G U}^{4}\right)^{-1 / 2}
$$

Hence, $\alpha \sim\left(\frac{128 \pi^{3} \tau^{2}}{90} \nu T_{G U}^{4}\right)^{1 / 2} e^{2 N_{e}} \approx 10^{62}$ and $\beta \approx 10^{-65}$ for a e-fold number $N_{e}=75$. Accordingly, unless for $\xi$ very close to zero, we may neglect the second term in (7). From the asymptotical expression of the $K_{0}$ Bessel function appearing in eq.(8) we may deduce the value of $\varpi$ (i.e. $\Lambda_{0}$ ) insuring a given number $N_{e}$ of e-fold :

$$
\varpi=2 N_{e}+\frac{1}{2} \ln \frac{2^{12} \pi^{2} \nu T_{G U}^{4}}{3}+\frac{1}{2} \ln \left[2 N_{e}+\frac{1}{2} \ln \frac{2^{12} \pi^{2} \nu T_{G U}^{4}}{3}\right] .
$$

This implies $\Lambda_{0}<<1$ for a large range of reasonable values of $N_{e}$, in particular $\Lambda_{0} \approx$ $1.4 \times 10^{-7}$ for $N_{e}=75$, what is compatible with the domain of validity of the model, while for $\Lambda_{0}=1 /\left(192 \pi^{2}\right)$, which corresponds to the assumption of thermal equilibrium between de Sitter temperature and Hawking temperature, we obtain $N_{e} \simeq 2400$.

According to the range of the values settled by the initial conditions, we may build approximate solutions of the perturbed equations. For instance, let us suppose that $\mathcal{R}$ scales with $\alpha$ as $\alpha^{K}$. From the perturbed equations, using the conservation of the entropy of the radiation as the cosmological constant goes to zero, we obtain $\mathcal{H} \sim \alpha^{K-1}$ and $\mathcal{T} \sim \alpha^{K-1 / 2}$. From the perturbed equations, it comes out that in this case $\dot{\mathcal{R}} \sim \frac{\mathcal{T}}{a} \sim \alpha^{K-1}$, which is negligible compared to $\frac{32 \pi}{3} \rho_{(\mathrm{rad} .)} a^{2} \mathcal{H} \sim \alpha^{K}$. Hence, the perturbed equations can be approximated by :

$$
\begin{aligned}
\dot{\mathcal{H}} & =\frac{\mathcal{R}}{a^{2}}, \\
\dot{\mathcal{R}} & =\frac{32 \pi}{3} \rho_{(\text {rad. })} a^{2} \mathcal{H}, \\
\dot{\mathcal{T}} & =\frac{n^{2}}{3} \frac{\mathcal{R}}{a},
\end{aligned}
$$


which can be integrated by quadratures. The general solution is :

$$
\begin{aligned}
\mathcal{H} & =\mathcal{H}_{i} \frac{Z\left(t_{i}\right)}{Z(t)}+\frac{\mu}{Z(t)} \int_{t_{i}}^{t} Z\left(t^{\prime}\right) d t^{\prime}, \\
\mathcal{R}(t) & =Z(t) \dot{\mathcal{H}}(t)=\mu Z(t)-\dot{Z}(t) \mathcal{H}(t), \\
\mathcal{T} & =\mathcal{T}_{i}+\frac{n^{2}}{3} \int_{t_{i}}^{t} \frac{\mathcal{R}\left(t^{\prime}\right)}{a\left(t^{\prime}\right)} d t^{\prime}
\end{aligned}
$$

with

$$
\mu=\frac{1}{Z\left(t_{i}\right)}\left(\mathcal{R}_{i}+\dot{Z}\left(t_{i}\right) \mathcal{H}_{i}\right)
$$

and $\mathcal{R}_{i}, \mathcal{H}_{i}, \mathcal{T}_{i}$ being the initial values of $\mathcal{R}, \mathcal{H}$ and $\mathcal{T}$.

On the other hand, if $\rho a^{3} \mathcal{H}$ can be neglected in front of $\mathcal{T}$, the system reduces to

$$
\frac{d \mathcal{R}}{d \zeta}=-\mathcal{T} \quad, \quad \frac{d \mathcal{T}}{d \zeta}=\frac{n^{2}}{3} \mathcal{R} \quad, \quad \dot{\mathcal{H}}=\frac{\mathcal{R}}{a^{2}}
$$

where $\zeta=\int_{t_{i}}^{t} \frac{d t^{\prime}}{a\left(t^{\prime}\right)}$. Its general solution is then

$$
\begin{aligned}
\mathcal{R} & =\mathcal{R}_{i} \cos \left(\frac{n}{\sqrt{3}} \zeta\right)-\frac{\sqrt{3}}{n} \mathcal{T}_{i} \sin \left(\frac{n}{\sqrt{3}} \zeta\right), \\
\mathcal{T} & =\mathcal{T}_{i} \cos \left(\frac{n}{\sqrt{3}} \zeta\right)+\frac{n}{\sqrt{3}} \mathcal{R}_{i} \sin \left(\frac{n}{\sqrt{3}} \zeta\right), \\
\mathcal{H} & =\mathcal{H}_{i}+\int_{t_{i}}^{t} \frac{\mathcal{R}\left(t^{\prime}\right)}{\tau Z\left(t^{\prime}\right)} d t^{\prime} .
\end{aligned}
$$

The initial time $t_{i}$, where the initial values of $\mathcal{R}, \mathcal{T}$ and $\mathcal{H}$ have to be considered, depend on the size of the perturbations. Today the temperature is of the order of $T_{0} \sim 2 \times$ $10^{-32}$, the cosmological observational data which are somehow free of astrophysical noises mainly concerns sizes $\lambda_{0}$ from $100 \mathrm{Mpc}$ up to $3.000 \mathrm{Mpc}$, i.e., $2 \times 10^{62} l_{P l}$ up to $6 \times 10^{63} l_{P l}$. At these scales the Universe can be considered as homogeneous. At the grand unification epoch, the size of these fluctuations are shortened by a factor $T_{0} / T_{G U}=2 \times 10^{-28}$. They must still be reduced by a factor $a\left(t_{i}\right) / a\left(t_{G U}\right)$ in order to obtain their values at the initial time. Moreover, the evolution of the fluctuations is given by equations (14, 15, 16) only once they grow faster than the microscopic interactions can propagate. It is only then that their behavior is no more dictated by the microphysics but only by the overall cosmological expansion. This implies that the evolution of perturbations with comoving wave numbers $n$ and present day size $\lambda_{0}$ will only be determined by the perturbation equations from an initial time $t_{i}(n)$ given by $\dot{a}\left(t_{i}(n)\right)=n$, i.e.,

$$
\dot{a}\left(t_{i}(n)\right)=\frac{2 \pi}{\lambda_{0}} \frac{T_{G U}}{T_{0}} e^{N_{e}} .
$$

This time $t_{i}(n)$ may be easily estimated by noting that $\varpi e^{-\xi_{G U}}$, and thus $\varpi e^{-\xi_{n}}$ (where $\xi_{i}(n)=t_{i}(n) / 2 \tau$ ), depends very slowly on the number of e-folds. Moreover, near 
$t_{i}(n)=0$ equation (38) easily may be linearized. Thus for each value of $\lambda_{\star}$, it is possible to determine a minimal number $N^{\min }\left(\lambda_{\star}\right)$ of e-folds such that fluctuations of size as $\lambda_{\star}$ today emerges from the horizon at $t=0$ :

$$
N^{\min }\left(\lambda_{\star}\right)=\ln \left[\frac{\lambda_{\star}}{4 \pi \tau} \frac{T_{0}}{T_{G U}} \ln \frac{\lambda_{\star} T_{0}}{4 \pi^{2} \tau T_{G U} \sqrt{\xi_{G U}}}\right]+1.93 \quad,
$$

and giving this number, we obtain from eq. (26), for a given number $N_{e}$ of e-folds,

$$
t_{i}\left(\lambda_{\star}\right) \approx 2 \tau \ln \left[\frac{\varpi\left(N_{e}\right)}{\varpi\left(N^{\min }\right)}\right]
$$

as the time where a fluctuation of size $\lambda_{\star}$ has emerged from the horizon. We restrict ourselves to positive values of $t_{i}$ (we do not know the physics before $t<0$ ). This implies that the minimum number of e-fold between $t=0$ and the beginning of the grand unification era we will consider is at least 75 .

The decay of the cosmological constant increases very rapidly the temperature (see fig. 1), but nevertheless we shall suppose that the density fluctuations are given by the laws of the equilibrium statistical mechanics [19]:

$$
\delta \rho\left(t_{i}, n\right)=\nu_{e f f}^{1 / 2} T^{5 / 2}\left(\frac{a\left(t_{i}(n)\right)}{n}\right)^{3 / 2} \frac{1}{a^{3}\left(t_{i}(n)\right)} \quad .
$$

The extra factor $a^{-3}\left(t_{i}(n)\right)$ is the jacobian that we have to introduce when we consider the Fourier transforms with respect to the comoving variables $n$ which are related to the physical momentum $n_{\text {phys }}$ at time $t_{i}$ by

$$
n_{\text {phys }}=\frac{n}{a\left(t_{i}\right)}=\frac{2 \pi}{\lambda_{\star}} \frac{T_{G U}}{T_{0}} \frac{e^{N_{e}}}{a\left(t_{i}\right)} .
$$

For consistency, we may check that the ratio of the proper energy density of such fluctuation to the temperature $\epsilon(n) / T_{i}(n)$ is approximatively equals to 1.8 when the fluctuation emerges from the horizon, the temperature $T_{i}(n)$ at this moment being of the order or $10^{-3}$. We may also check that these values are very few sensitive to the values of $\tau$ and $N_{e}$. Moreover, we also assume the initial spectrum of $\theta\left(t_{i}(n)\right)$ and $\dot{h}\left(t_{i}(n)\right)$ being given by classical physics, i.e., they are not affected by the overall expansion of the Universe. Using standard arguments [19, 20], we obtain

$$
\begin{aligned}
& \theta\left(t_{i}, n\right) \sim \nu_{\text {eff }}^{1 / 2} T_{i}^{-3 / 2}(n) n^{-1 / 2} a\left(t_{i}(n)\right)^{-5 / 2}, \\
& \dot{h}\left(t_{i}, n\right) \sim \nu_{\text {eff }}^{1 / 2} T_{i}^{5 / 2}(n) n^{-5 / 2} a\left(t_{i}(n)\right)^{-1 / 2} .
\end{aligned}
$$

Hence, $\mathcal{R}\left(t_{i}, n\right)=Z^{2}\left(t_{i}(n)\right) \delta \rho\left(t_{i}, n\right), \mathcal{T}\left(t_{i}, n\right) \sim n \mathcal{R}\left(t_{i}, n\right)$ and $\mathcal{H}\left(t_{i}, n\right) \sim \mathcal{R}\left(t_{i}, n\right) / n a\left(t_{i}, n\right)$ The second relation means that the longitudinal mode $h\left(t_{i}, p\right)$ is determined by the gravitational fluctuations, with a characteristic time of the order of the size of the fluctuations considered (of course, one also could ask about the metaphysical content of these considerations, that we adopt in the absence of a better scenario). 
With these assumptions, it is possible to verify that at initial times $\mathcal{T} / a$ is about $2-5 \times 10^{3}$ times greater than $\frac{32 \pi}{3} \rho_{(\mathrm{rad} .)} a^{2} \mathcal{H}$ :

$$
\frac{32 \pi}{3} \rho_{i} a_{i}^{3} \frac{\mathcal{H}_{i}}{\mathcal{T}_{i}} \approx 8 \pi \frac{a^{2}\left(t_{i}\right) \rho_{(\text {rad. })}\left(t_{i}\right)}{n^{2}} \approx \frac{3}{16 \tau^{2} n^{2}} \varpi e^{-\xi_{i} / 2} e^{\varpi\left(1-e^{-\xi_{i}}\right)}<<1
$$

a result again almost insensitive to the values of $N_{e}$ and $\tau$. As a consequence, during a first short period of time we may approximate the system of the perturbation equations by the eqs (27,28,29). Hence, one verify that the functions $\mathcal{H}, \mathcal{R}$ and $\mathcal{T}$ given by eqs $(35,36,37)$ change little in the beginning of the evolution of perturbations. But the ratio between the two terms in the right hand side of (15) behaves as:

$$
\frac{32 \pi}{3} \rho_{(\text {rad. })} a^{3} \frac{\mathcal{H}}{\mathcal{T}} \sim 8 \pi \frac{\rho_{(\text {rad. })}(t) a^{3}(t)}{n^{2} a\left(t_{i}\right)}
$$

and increases very quickly. Hence, for values of $\xi$ very near $\xi_{i}$, the system will be in the domain of validity of the first approximate solution (30,31). Taking into account the uncertainties on the the parameters of the problem (initial spectrum, precise duration of validity of the perturbed equations, etc.), we can adopt them as solutions of the perturbed equations. From these considerations, it comes out that at the grand unification temperature, the density contrast $\delta_{G U}=\left|\delta \rho\left(t_{G U}\right) / \rho_{G U}\right|$ is given by

$$
\delta_{G U}=\frac{\mathcal{R}\left(t_{G U}\right)}{\rho_{G U} Z_{G U}^{2}} \approx \frac{32 \pi}{3} \frac{\mu e^{2 N_{e}}}{\alpha^{2}},
$$

and all dependence on the wave number $n$ is contained in the value of the integration constant $\mu$. This constant is given by the sum of two terms (see eq. (33), but the first one $Z\left(t_{i}\right) \delta \rho_{i}$ is significantly more important than the second one. Hence, the spectrum index depends only on the initial spectrum of density perturbations and on the dates where, for a given size $\lambda_{0}$, the fluctuations emerge from the horizon. As the temperature $T\left(t_{i}, n\right)$ is almost constant during the interval of time where the relevant perturbations $\left(100 \mathrm{Mpc}<\lambda_{0}<3000 \mathrm{Mpc}\right)$ cross the horizon while $a\left(t_{i}(n)\right) \div n$ we immediately obtain the spectral index

$$
n_{s}=4+\frac{d}{d \ln n} \ln \left[(\delta \rho(n) / \rho)^{2}\right] \approx 2
$$

\section{Conclusions}

In this work, we have studied an inflationary model that joins the radiative regime by a second order phase transition. This model is inspired in an inflationary primordial scenario where the transition from a de Sitter phase to a radiative phase occurs due to the evaporation of primordial black holes. It must be considered as a source of a possible alternative to the usual inflationary models, and not as a definitive model. Indeed, the underlined physics leading to the background geometry is phenomenological and asks for more deep fundamental investigations. It offers, however, the conceptual advantage of making no appeal to a self-interacting scalar field. The kinetics of the phase transition 
asks also for a more rigorous argumentation. However, we believe our results not to be dependent on the details of the of the model as the spectral index obtained is very little sensible to the values of $N_{e}$ and $\tau$.

Moreover, we have introduced hypothesis on the nature of the initial spectrum of the fluctuations. In particular, we employed a thermal spectrum for the fluctuations as they emerge from horizon. Notice that, from the conceptual point of view, the spectrum more delicate is that of the $h$ but this quantity does not play a significative role in the computation of the spectral index, since the gravitational fluctuations is dominated by those, much more important, fluctuations of matter, which is governed by $\mathcal{R}$. The nature of the spectrum of $\delta \rho$ affects the spectral index essentially by the function $a\left(t_{i}\right) \sim n$ which appears in the jacobian of density fluctuation defining $\delta \rho_{i}$ and on the behavior of $Z\left(t_{i}, n\right)$, as appears in the expression for the initial conditions. Of course the obtained value of the spectral index is not in agreement with observational data. However here we have only discussed scalar fluctuations; it remains to see if tensorial fluctuations cannot reconcile the model with the expected Harrison-Zeldovitch spectra. But such an analysis reopen the question of the choice of the initial spectrum of the gravitational perturbations, a question whose answer, in our opinion is beyond our present knowledge of physics.

\section{ACKNOWLEDGMENTS}

The authors thank Jérôme Martin for fruitful discussions and CAPES (Brazil) for partial financial support. Ph. S. thanks Robert Brout, François Englert and Marianne Rooman for numerous enlightening discussions about many aspects of the study performed here, Jean Bricmont for noticing him ref. [16] and CAPES (Brazil) for partial financial support. He also thanks the Departamento de Física of UFES for its warm hospitality.

\section{References}

[1] A. Friedmann, Z. Phys. 10, 377(1922), G. Lemaître, Ann. Soc. Sci. Bruxelles, I A53, 51(1933); H. P. Robertson, Astrophys. J. 82, 248(1935); A. G. Walker, Proc. R. Soc. Edinburgh 52, 345(1932).

[2] A. H. Guth, Phys. rev. D 23(1981) 347, S. K. Blau and A.H. Guth, in 300 years of gravitation, edited by S. Hawking and W. Israel, Cambridge University Press, Cambridge(1987).

[3] S.W. Hawking and G.F.R. Ellis, The large scale structure of space-time, Cambridge University Press, Cambridge (1973);

[4] R. Brout, F. Englert and E. Gunzig, Ann. Phys. (NY) 115, 78(1978); Gen. Rel. Grav. 10, 1(1979).

[5] E.P. Tryon, Nature 246, 396(1973). 
[6] R. Brout, F. Englert and Ph. Spindel, Phys. Rev. Lett. 43, 417(1979); Phys. Rev. Lett. 43, 890(1979).

[7] R. Brout, F. Englert, J.-M. Frère, E. Gunzig, P. Nardone, C. Truffin and Ph. Spindel, Nucl. Phys. 107B,361(1981).

[8] Ph. Spindel, Phys. Lett. 107B, 361(1981).

[9] Ph. Spindel and P. Vandepeutte, Numerical investigations in cosmogenesis, in Proceedings of the 26th Liège International Astrophysical Colloquium, Université de Liège(1986).

[10] F. Englert, Primordial inflation, in Proceedings of the Erice International School on "Basics and Highlights in Fundamental Physics"(1999).

[11] A. Casher and F. Englert, Phys. Lett. B104, 117(1981).

[12] J.S. Dowker and R. Critchley, Phys. Rev. D12, 327(1976).

[13] Ph. Spindel, Phys. Rev. D37, 2092(1988).

[14] I. Antoniadis and E. Mottola, J. Math. Phys. 32(1991).

[15] R. Brout and Ph. Spindel, Phys. Lett. B320, 241(1999).

[16] B. Russell, My Philosophical Development, London: Routledge (1995 [1959, page 164]).

[17] J. Martin, A. Riazuelo, M. Sakellariadou Phys. Rev. D 61 (2000) 083518, astro$\mathrm{ph} / 9904167$.

[18] J. Martin and R. H. Brandenberger, The trans-planckian problem of inflationary cosmology, hep-th/0005209; J. C. Niemeyer, R. Parentani Trans-Planckian dispersion and scale-invariance of inflationary perturbations, astro-ph/0101451.

[19] L. Landau and E. Lifshitz, Mécanique statistique, Mir, Moscou(1967).

[20] L. Landau and E. Lifshitz, Mécanique des fluides, Mir, Moscou (1971). 